\title{
Spectral Analysis of Synchronization in Mobile Networks
}

\author{
Naoya Fujiwara*, Jürgen Kurths ${ }^{*, \dagger}$ and Albert Díaz-Guilera*** \\ *Potsdam Institute for Climate Impact Research (PIK), 14473 Potsdam, Germany \\ ${ }^{\dagger}$ Institute for Complex Systems and Mathematical Biology, University of Aberdeen, UK \\ ** Departament de Física Fonamental, Universitat de Barcelona, 08028 Barcelona, Spain
}

\begin{abstract}
We here analyze a system consisting of agents moving in a two-dimensional space that interact with other agents if they are within a finite range. Considering the motion and the interaction of the agents, the system can be understood as a network with a time-dependent topology. Dynamically, the agents are assumed to be identical oscillators, and the system will eventually reach a state of complete synchronization. In a previous work, we have shown that two qualitatively different mechanisms leading to synchronization in such mobile networks exist, namely global synchronization and local synchronization, depending on the parameters that characterize the oscillatory dynamics and the motion of the agents [1]. In this contribution we show that the spectral pattern differs between the two synchronization mechanisms. For global synchronization the spectrum is flat, which means that all eigenmodes contribute identically. For local synchronization, instead, the synchronization dynamics is determined mostly by the eigenmodes whose eigenvalues are close to zero. This result suggests that the global synchronization mechanism achieves fast synchronization by efficiently using the fast decaying eigenmodes (larger eigenvalues).
\end{abstract}

Keywords: Mobile network, Synchronization

PACS: $89.75 . \mathrm{Hc}, 05.45 . \mathrm{Xt}$

\section{INTRODUCTION}

Starting already with Huygens in 1665 , synchronization of autonomous agents is a paradigmatic example of emergence $[2,3]$. Recent development of the theory of complex networks enables us to study synchronization on intricate patterns of interaction [4, 5]. However, there are not enough studies for synchronization of networks with time evolving topologies $[6,7,8,9]$. In particular, there is a particular class of such evolving networks, whose nodes (agents) move around but interact only with nearby agents $[10,11,12]$. We name such systems mobile networks. There are many examples where synchronization in mobile networks is crucial: chemotaxis [13], mobile ad hoc networks [14], wireless sensor networks [15], and the expression of segmentation clock genes [16].

In the literature, prior studies on synchronization in evolving networks are concentrated so far on two special cases. On the one hand, when the network topology changes in a fast manner, a good approximation is to consider a network where the connections are represented by the probability of pairs of agents being connected. This is the so-called fast-switching approximation (FSA) [17, 18, 19, 20]. On the other hand, when the population of agents is dense and arranged in a ring, the Fokker-Planck equation formalism has been used to describe the synchronization transition and the emergence of a spatial structure [21]. Recently, we proposed a general framework for mobile oscillator networks where agents perform random walks in a two-dimensional (2D) plane [1]. We have shown [1] that FSA fails when the time scale of local synchronization is shorter than that of the topology change due to the motion of the agents. Those time scales arise due to the interplay between instantaneous network topology, agent motion, and interaction rules.

The development of analytical methods for studying synchronization of mobile networks is crucial for the understanding of the dynamical properties of the system [1]. Here we propose a method based on the spectral decomposition of the time-dependent Laplacian matrix. We show that the obtained spectral pattern depends on whether the FSA holds, and hence the different mechanisms give rise to different spectral patterns.

The organization of the paper is as follows. In the next section, we summarize the model and results of our previous work [1]. Then we propose a spectral method to analyze synchronization in mobile networks, and discuss the statistical properties of different dynamical regimes.

Numerical Analysis and Applied Mathematics ICNAAM 2011

AIP Conf. Proc. 1389, 1015-1018 (2011); doi: 10.1063/1.3637782

(C) 2011 American Institute of Physics 978-0-7354-0956-9/\$30.00 


\section{MODEL SETTINGS}

First we describe our model which has been introduced in Ref. [1]. This is a model of mobile agents moving in a 2D plane of size $L$ with periodic boundary conditions. The state of each agent is characterized by a phase variable. We assume a type of interaction that makes that phases approach one another, but only for nearby agents within a certain spatial distance $d$. For instance, this stands for agents holding wireless devices interacting through a Bluetooth protocol.

In this model the time evolution of the $i$ th agent position of the $\left(x_{i}(t), y_{i}(t)\right)$ is

$$
\begin{aligned}
& x_{i}\left(t_{k}+\Delta t\right)=x_{i}\left(t_{k}\right)+v \Delta t \cos \xi_{i}\left(t_{k}\right) \bmod L \\
& y_{i}\left(t_{k}+\Delta t\right)=y_{i}\left(t_{k}\right)+v \Delta t \sin \xi_{i}\left(t_{k}\right) \bmod L,
\end{aligned}
$$

where $\Delta t \leq \tau_{M}$. This comes from the fact that the angle of the $i$ th agent's motion is $\xi_{i}\left(t_{k}\right) \in[0,2 \pi]$, changing randomly at discrete time steps $t_{k}\left(t_{k+1}-t_{k}=\tau_{M}\right)$.

On the other hand, the time update of the agent phases is given by

$$
\varphi_{i}\left(t+\tau_{P}\right)=\varphi_{i}(t)+\sigma \sum_{j, d_{i j}<d} \sin \left[\varphi_{j}(t)-\varphi_{i}(t)\right],
$$

where $d_{i j}=\sqrt{\left(x_{i}-x_{j}\right)^{2}+\left(y_{i}-y_{j}\right)^{2}}$. Note that we consider discrete time updates with a time step $\tau_{P}$. In our previous paper, we found that the average phase difference $\sqrt{\frac{2}{N(N-1)} \sum_{j<k}\left[\varphi_{j}(t)-\varphi_{k}(t)\right]^{2}}$ decays exponentially in time after the initial transient with a characteristic time $T$ and reaches a state of complete synchronization [1].

When the phase difference is small, the linearized equation

$$
\varphi_{i}\left(t+\tau_{P}\right)=\varphi_{i}(t)-\sigma \sum_{j=1}^{N} L_{i j}(t) \varphi_{j}(t)
$$

approximately describes the dynamics of the phases. Here, the time dependent Laplacian matrix $L(t)$ is introduced as $L_{i j}(t)=\left[k_{i}(t) \delta_{i j}-c_{i j}(t)\right]$, and $c_{i j}(t)=1$ if $d_{i j}<d(i \neq j)$ and $c_{i j}(t)=0$ otherwise. $k_{i}(t)$ represents the degree of the $i$ th node, i.e. the number of oscillators that are around $i$ within a range $d$. When the agents move fast enough and the network topology changes very fast, we can apply the FSA [17, 18, 19, 20], which consists in replacing the entries in the connectivity matrix in Eq. (3) by its time average. By this approximation the characteristic time $T_{F S}$ is estimated as

$$
T_{F S}=-\tau_{P} / \log [1-\sigma(N-1) \rho],
$$

where $\rho=\pi d^{2} / L^{2}$, for $d \leq L / 2$.

Our numerical simulations show that there are two different mechanisms leading to complete synchronization, namely global and local synchronization, depending on the parameters of the system [1]. For global synchronization (Fig. 1(a)), all agents approach complete synchronization almost at the same rate. In this case, the FSA describes very well the characteristic time $T$. Global synchronization is observed in the parameter range where the time scale of topology change is much shorter than that of local synchronization inside an isolated cluster. On the other hand, when the latter is much shorter than the former, we observe important deviations from the FSA (Fig. 1(b)). In this case, we get locally synchronized clusters in the intermediate time regime. Such clusters grow up, and eventually, complete synchronization is attained as well. Local synchronization is also observed when $d$ is large enough because the whole network forms a single connected component (single cluster local synchronization, Fig. 1(c)).

A detailed analysis of the linear approximation is available by decomposing Eq. (3) into normal modes $\theta_{l}(t)$ corresponding to the eigenvalues $\lambda_{l}(t)$ of $L(t) . \varphi_{j}$ and $\theta_{l}$ are transformed by the orthogonal matrix $U_{j l}(t)$ such as $\varphi_{j}(t)=\sum_{l=1}^{N} U_{j l}(t) \theta_{l}(t)$. The time evolution of $\theta_{l}$ can be described as

$$
\theta_{l}\left(t+\tau_{P}\right)=\sum_{m=1}^{N} O_{l m}(t)\left[1-\sigma \lambda_{m}(t)\right] \theta_{m}(t)
$$

by multiplying Eq. (3) by the transpose $U_{l i}^{T}\left(t+\tau_{P}\right)$ on the left. Note that $O_{l m}(t) \equiv \sum_{i} U_{l i}^{T}\left(t+\tau_{P}\right) U_{i m}(t)$ is also orthogonal. Hence the time evolution of the normal mode after $n$ time steps can be written in terms of the product of matrices as $\theta_{l_{n}}\left(t+n \tau_{P}\right)=\prod_{q=0}^{n-1}\left[\sum_{l_{q}=1}^{N} O_{l_{q+1} l_{q}}\left(1-\sigma \lambda_{l_{q}}\right)\right] \theta_{l_{0}}(t)$, where $l_{q}$ denotes the suffix corresponding to an eigenmode at time $t+q \tau_{P}$. This matrix product consists of two parts: the transformation of the normal modes of instantaneous networks by $O_{l_{q+1} l_{q}}$, and the decay of each eigenmode in $\tau_{P}$ by $\left(1-\sigma \lambda_{l_{q}}\right)$. 

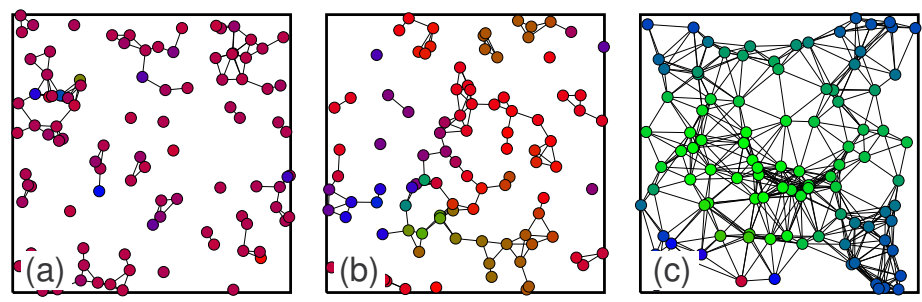

FIGURE 1. Phase distribution of different synchronization mechanisms for (a) global synchronization, (b) multiple cluster local synchronization, and (c) single cluster local synchronization cases. The colors represent the phases in $[0,2 \pi]$.

\section{STATISTICAL PROPERTIES OF THE NORMAL MODES OF THE MATRIX PRODUCT}

In this section, we extend Eq. (5), derived in Ref. [1], and propose a spectral analysis in order to characterize the asymptotic behavior of the system. Since $O_{l m}$ in Eq. (5) is orthogonal, we get the time evolution of the norm $\theta^{2}(t)=\sum_{l=1}^{N} \theta_{l}^{2}(t)$ as $\left(\bar{\theta}_{l} \equiv \theta_{l} / \theta\right)$

$$
\begin{aligned}
\theta^{2}\left(t+\tau_{P}\right) & =\theta^{2}(t)\left[\sum_{l}\left(1-\sigma \lambda_{l}(t)\right)^{2} \bar{\theta}_{l}^{2}(t)\right] \\
\sum_{l=1}^{N} \bar{\theta}_{l}^{2} & =1 .
\end{aligned}
$$

When the number of agents and hence the number of eigenmodes is large, we can assume a continuous distribution of eigenvalues in the steady state $f(\lambda)$, with $\int d \lambda f(\lambda)=1$. Then we can analyze the statistical properties of $\bar{\theta}(\lambda)$ as a function of the eigenvalue $\lambda$. Introducing $\langle A\rangle$ as the long-time average of $A$, we get $N \int d \lambda f(\lambda)\left\langle\bar{\theta}^{2}(\lambda)\right\rangle=$ 1 from Eq. (7). Note that the factor $N$ appears because there are $N$ eigenmodes in $L(t)$. Since the approach to synchronization is exponential with a characteristic time $T$, the ratio between the norms at two consecutive updates will be $\left\langle\theta^{2}\left(t+\tau_{P}\right) / \theta^{2}(t)\right\rangle=e^{-2 \tau_{P} / T}$, which leads to

$$
2 \tau_{P} / T=-\log \left[N \int d \lambda f(\lambda)(1-\sigma \lambda)^{2}\left\langle\bar{\theta}^{2}(\lambda)\right\rangle\right] .
$$

This equation shows that if $\left\langle\bar{\theta}^{2}(\lambda)\right\rangle$ takes a large value for a large $\lambda$, fast synchronization is achieved. When $\sigma$ is small enough, we can expand the logarithm in $\sigma$ and, taking the lowest order term, $T$ can be approximated to

$$
\frac{\tau_{P}}{T} \approx N \sigma \int d \lambda f(\lambda) \lambda\left\langle\bar{\theta}^{2}(\lambda)\right\rangle
$$

Figures 2 (a), (b), and (c) show the results for global synchronization, multiple cluster local synchronization, and single cluster local synchronization cases, respectively. These results clearly show that spectral pattern is substantially different for global or local synchronization.

For global synchronization (Fig. 2(a)), the distribution is flat. In this case, the network topology changes much faster than the time scale required for local synchronization. Therefore, $\theta_{m}$ with small $\lambda_{m}$ is distributed to $\theta_{l}$ with larger $\lambda_{l}$ via $O_{l m}$ term in Eq. (5). This is how the flat distribution is obtained for the global synchronization case. Using the equality $N \int d \lambda f(\lambda) \lambda=(N-1) \rho$, given that the average eigenvalue of the Laplacian matrix is the average degree, Eq. (9) can be written as

$$
\frac{\tau_{P}}{T}=\sigma(N-1) \rho
$$

which is identical to Eq. (4) to the lowest order in $\sigma$. This result suggests that the FSA is appropriate when the spectrum is flat.

For local synchronization (Figs. 2(b) and (c)), the time scale of topology change is larger than that of synchronization inside a cluster. This implies that normal modes with larger $\lambda$ decay faster than those with smaller $\lambda$, and transport 
between them does not take place easily. Therefore $\left\langle\bar{\theta}^{2}(\lambda)\right\rangle$ is larger for normal modes with smaller $\lambda$. The time $T$ required for complete synchronization is larger when the local mechanism dominates than in the case of global synchronization, because smaller normal modes do not contribute to the decay.

To summarize, we conclude that synchronization achievement is faster for global synchronization than for local synchronization, because modes with larger $\sigma \lambda$ contribute to synchronization achievement more for the flat spectrum of the global synchronization, as it clearly deduced from Fig. 2. Besides, Fig. 2 also implies that the FSA is a very efficient mechanism for complete synchronization. At the same time, this result suggests the existence of a more efficient complete synchronization mechanism: if the larger eigenmode is dominant, faster synchronization is achieved.
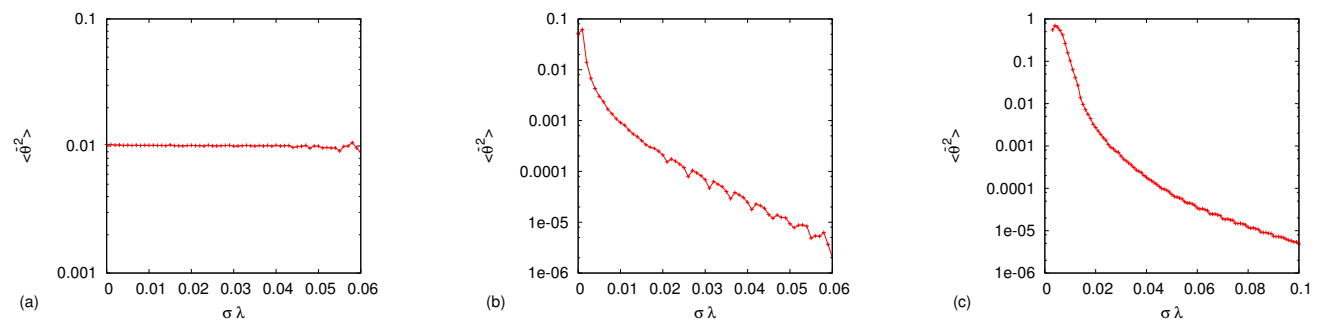

FIGURE 2. Dependence of the spectrum $\left\langle\bar{\theta}^{2}(\lambda)\right\rangle$ on the eigenvalue $\lambda$ of the Laplacian matrix for (a) global synchronization for $d=20$ and $\tau_{P}=10$, (b) multiple cluster local synchronization for $d=20$ and $\tau_{P}=0.01$, and (c) single cluster local synchronization for $d=40$ and $\tau_{P}=0.01$. Parameter values are the same as in the corresponding panels in Fig. 1. Other parameters are $N=100$, $L=200, v=10, \sigma=0.005$, and $\tau_{M}=1$.

\section{ACKNOWLEDGMENTS}

NF and JK are grateful for financial support from WGL (project ECONS) and FET Open project SUMO (grant agreement 266722). AD-G acknowledges financial support from Spanish MCINN (grants FIS2006-13321, FIS200913730, PR2008-0114) and Generalitat de Catalunya (grant 2009SGR00838).

\section{REFERENCES}

1. N. Fujiwara, J. Kurths, and A. Díaz-Guilera, Phys. Rev. E 83, 025101(R) (2011).

2. A. Pikovsky, M. Rosenblum, and J. Kurths, Synchronization, Cambridge University Press, Cambridge, UK, 2001.

3. Y. Kuramoto, Chemical oscillations, waves, and turbulence, Springer-Verlag, New York, NY, USA, 1984.

4. S. Boccaletti, V. Latora, Y. Moreno, M. Chavez, and D.-U. Hwang, Phys. Rep. 424, 175-308 (2006).

5. A. Arenas, A. Díaz-Guilera, J. Kurths, Y. Moreno, and C. Zhou, Phys. Rep. 469, 93-153 (2008).

6. M. L. Sachtjen, B. A. Carreras, and V. E. Lynch, Phys. Rev. E 61, 4877-4882 (2000).

7. R. Olfati-Saber, J. A. Fax, and R. M. Murray, P. IEEE 95, 215-233 (2007).

8. J.-P. Onnela, J. Saramaki, J. Hyvonen, G. Szabo, D. Lazer, K. Kaski, J. Kertesz, and A. L. Barabasi, Proc. Natl. Acad. Sci. USA 104, 7332-7336 (2007).

9. M. Valencia, J. Martinerie, S. Dupont, and M. Chavez, Phys. Rev. E 77, 050905(R) (2008).

10. A. Buscarino, L. Fortuna, M. Frasca, and A. Rizzo, Chaos 16, 015116 (2006).

11. H. G. Tanner, A. Jadbabaie, and G. J. Pappas, "Stable flocking of mobile agents part I: dynamic topology," in Decision and Control, 2003. Proceedings. 42nd IEEE Conference on, 2003, vol. 2, pp. 2016-2021 Vol.2.

12. J. Buhl, D. J. T. Sumpter, I. D. Couzin, J. J. Hale, E. Despland, E. R. Miller, and S. J. Simpson, Science 312, 1402-1406 (2006).

13. D. Tanaka, Phys. Rev. Lett. 99, 134103 (2007).

14. K. Römer, Proceedings of the 2nd ACM international symposium on Mobile ad hoc networking \& computing pp. 173-182 (2001).

15. F. Sivrikaya, and B. Yener, Network, IEEE 18, 45-50 (2004).

16. K. Uriu, Y. Morishita, and I. Yoh, Proc. Natl. Acad. Sci. USA 107, 4979-4984 (2010).

17. I. V. Belykh, V. N. Belykh, and M. Hasler, Physica D 195, 188-206 (2004).

18. M. Frasca, A. Buscarino, A. Rizzo, L. Fortuna, and S. Boccaletti, Phys. Rev. Lett. 100, 044102 (2008).

19. M. Porfiri, D. J. Stilwell, E. M. Bollt, and J. D. Skufca, Physica D: Nonlinear Phenomena 224, 102 - 113 (2006).

20. D. J. Stilwell, E. M. Bollt, and D. G. Roberson, SIAM J. App. Dyn. Syst. 5, 140-156 (2006).

21. F. Peruani, E. M. Nicola, and L. G. Morelli, New J. Phys. 12, 093029 (2010). 